\title{
Model of Experience for Public Organisations with Staff Mobility
}

\author{
Jacek Kitowski ${ }^{1,2}$, Krzysztof Krawczyk ${ }^{1}$, Marta Majewska ${ }^{1}$, \\ Mariusz Dziewierz ${ }^{1}$, Renata Słota ${ }^{1,2}$, Simon Lambert ${ }^{3}$, Alistair Miles ${ }^{3}$, \\ Alvaro Arenas ${ }^{3}$, Ladislav Hluchý ${ }^{4}$, Zoltan Balogh ${ }^{4}$, Michal Laclavik ${ }^{4}$, \\ Sabine Delaître $^{5}$, Gianni Viano ${ }^{6}$, Simona Stringa ${ }^{6}$, and Pasqualino Ferrentino ${ }^{6}$ \\ 1 ACC CYFRONET-AGH, ul. Nawojki 11, Cracow, Poland \\ 2 Institute of Computer Science AGH-UST, al. Mickiewicza 30, Cracow, Poland \\ 3 CLRC Rutherford Appleton Laboratory, Chilton, Didcot, OX11 0QX, UK \\ 4 Institute of Informatics of SAS, Dubravska cesta9, 84507 Bratislava, Slovakia \\ 5 Sadiel SA, C/Isaac Newton,s/n. Edificio Sadiel-Isla de la Cartuja, Sevilla, Spain \\ 6 Softeco Sismat SpA, Via De Marini 1, Torre WTC, 16149 Genova, Italy
}

\begin{abstract}
Public organisations with a high rate of staff rotation lose their valuable asset - experience of employees. Mobile employees, who have to face duties at new positions, would find computer assistance helpful in efficient inception. The idea to support current work of employees on the basis of gathered experience in past activities is driving the Pellucid project (IST-2001-34519). To serve experience-base assistance to the employees it is necessary to have experience modelled first. The comprehensive model containing user profiles, which defines experience in the scope of organisation, is described in the paper. The representation of the business process items and employees expertise level in the domain of organisation's activity is created on the basis of the organisation model. To represent experience an active hint approach is provided. Active hints are presented to employees spontaneously and on demand according to the their level of competence. Furthermore, the methods of experience gathering are discussed in detail on the basis of the platform development in the Pellucid project pilot sites.
\end{abstract}

\section{Introduction}

Nowadays, one of the most valuable assets of organisations is experience of their employees. Organisations with high level of staff mobility suffer significant loss if experience is regarded only as an ability, which belongs to the employees. If the experience was accumulated by the organisation, e.g. in the computer system, it could be many times reused.

The Pellucid project [1] is deriving from the idea of capturing and reusing of experience. Assistance to the novice employees at the initial stage of their work based on past gathered experience may be done by a dedicated software platform [23:4]. The Pellucid functionality is not limited to the accumulation of experience only. A desired situation for the employees is to get an adequate 
and accurate piece of experience just in time. Such an adaptation of experience to the individual needs is especially important in the case of mobile employees. Novices find helpful a selection of experience items that allow them simply progressing. The Pellucid platform is designed to serve employee assistance in form of experience items spontaneously or on demand.

In the paper a model of the uniform experience management for public organisation is described. The model is general in its assumptions. All, medium and large-size, organisations with semi-structured business administration process, and with knowledge-intensive activities performed by the employees, are addressed. At the initial stage three installations will be provided for project pilot sites.

In Sect.2 the state of the art is described. Models created in the stage of system analysis and design are presented in Sects.3.5. together with brief presentation of the methodology. A description of the domain modeling for the pilot organisations is given in Sect.6. The models are used for organisation ontology specification, which is discussed in Sect.7. Finally, the proposed approach to the experience acquisition is introduced in Sect.8. Conclusions in Sect.9 are finishing the paper.

\section{State of the Art}

Knowledge management for public sector organisations has become very important issue during the last decade. Several projects supporting public sector have been developed so far or are still in development. Scientific community tries to support employees by providing a service called an Organisational Memory [56]. Such a memory is treated as a collector of all kinds of organisational knowledge and is specially designed for the reuse in the future. DECOR [7, FRODO 8, KnowMore 9] projects deal with such a knowledge repository.

Information and knowledge stored within an organisational memory is often described with the help of the ontology [510]. The ontological approach has been found especially useful in the knowledge management systems $[2,7,8,9,11]$.

Problems rising within the public sector organisations, deriving from the nature of their business process. They can be characterized and described, but cannot be formalized fully. These types of processes are often called semistructured [12. Extensions to the traditional business process are necessary. Some approaches integrate the workflow management system with delivering of knowledge 21314. In such a solution the workflow management system gives the description of the context. On the basis of that context knowledge and/or information is delivered to the user. The collaborative nature of knowledge intensive tasks 15] and the distribution of several knowledge sources entails the use the intelligent software agents in this area 81617 18. Modern agent based systems are realized implementing object oriented programming languages, like Java, C++ or Smalltalk and use distributed objects environments, e.g., CORBA [19], DCOM [20], Voyager [21]. 


\section{Organisation Model}

The Pellucid system modelling has been highly influenced by the CommonKADS methodology for developing knowledge-based systems [22]. In CommonKADS, a development of a system entails constructing a set of engineering models of problem solving behaviour in its concrete organisation and application contexts. This modelling concerns not only expert knowledge, but also various characteristics of how that knowledge is embedded and used in the organisational environment. We have followed a similar approach in the Pellucid project, borrowing some components from the CommonKADS methodology.

The CommonKADS organisation model describes the organisation in a structured, systems-like way, by representing aspects such as an organisation structure, processes, staff, and resources. All these aspects were taken into account in our organisation model. The main CommonKADS model specifies knowledge and reasoning requirements of the prospective system. It deals with the domain-specific knowledge and information used within the application, and the task/inference knowledge, which express application's goals and its realisation through a decomposition into subtasks and ultimately inferences. We have followed a similar approach in the Pellucid experience model. The working context deals with domain-specific characteristics of an organisation and active hints formalize the inferences needed in helping employees.

The ORGANISATION is defined by a structure, employees and goals oriented toward serving citizens. The public sector organisation achieves its goals by settling a set of AFFAIRS (see Fig. 1). Each affair engages EMPLOYEES. The employees work to accomplish ACTIVITIES, which are necessary to settle the whole affair. Each activity requires employee's SKILLS to be performed efficiently. A set of skills that the employee possesses does not necessarily contains all skills needed to perform the specific activity. An employee is chosen to work with affair on the basis of a ROLE played in the organisation. However, the role of employee may change dynamically according to current needs. The employee experiencing frequent changes of his/her role in the organisation is called a mobile employee.

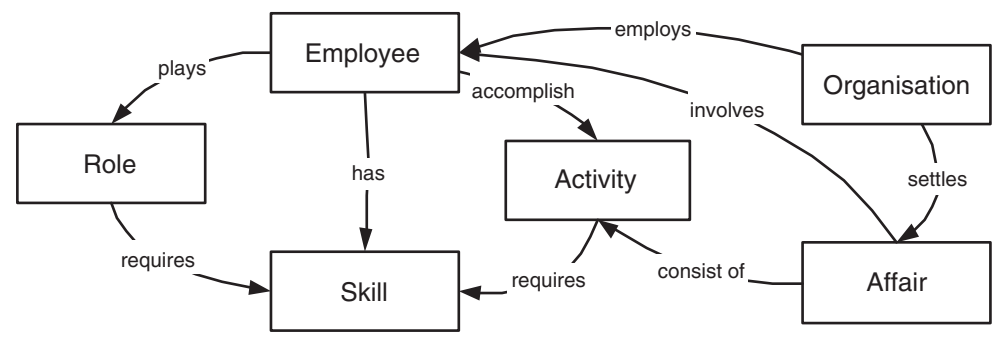

Fig. 1. Fragment of the organisation model 


\section{Experience Model}

In the Pellucid system, EXPERIENCE is defined as an ability to perform an ACTION according to the given CONTEXT (see Fig. 2). The context is composed of tasks to be performed, actors to cooperate and resources to be exploited. It should describe all circumstances in which a particular activity is taken. The accomplishment of activity requires a PROBLEM to be solved. The problem is described in terms of the domain specific ontology. Moreover, some external factors may influence the way of activity accomplishment such as RESOURCES that are involved in the problem solution, both the HUMAN beings and elements of an INFRASTRUCTURE, e.g., Workflow Management System or Workflow Tracking System.

The idea of an active user support by knowledge delivery, triggered according to the context in a workflow, was developed by the DECOR project 7]. The Pellucid project has borrowed the idea of an "active hints" as conveyors of experience, and is working it out in a somewhat different direction. Active hints are regarded as suggestions to the user to perform some actions that will assist his/her current activity in the process instance. An active hint is a special element of knowledge which consists of an action, a resource and a justification (cf. Fig. 2), together with a context against which the current working context is matched and which will trigger a presentation of the hint. This context covers both the context in the work process (starting or completing a task, opening a document) as well as a domain-specific representation (relevant similarity of the current case to prior cases). An additional context is the characteristics of the employee (whether experienced or novice) at the most basic level.

The part of the hint called action is what the employee is suggested to do, e.g., to examine some documents, contact a person or use a template. The resource is the "subject" variable of the action. The justification presents a reason why the hint is being suggested. This model of active hints is very general, since

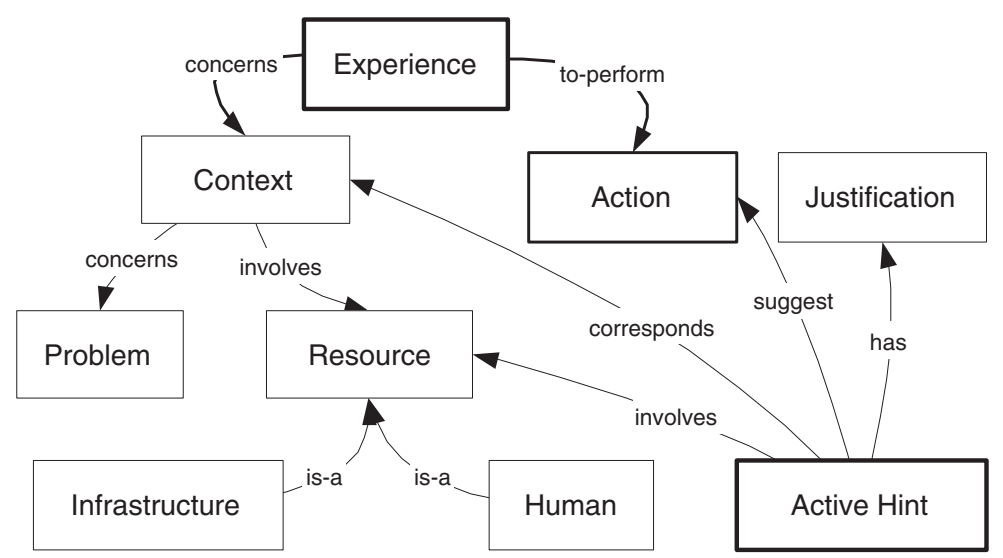

Fig. 2. Model of Experience 


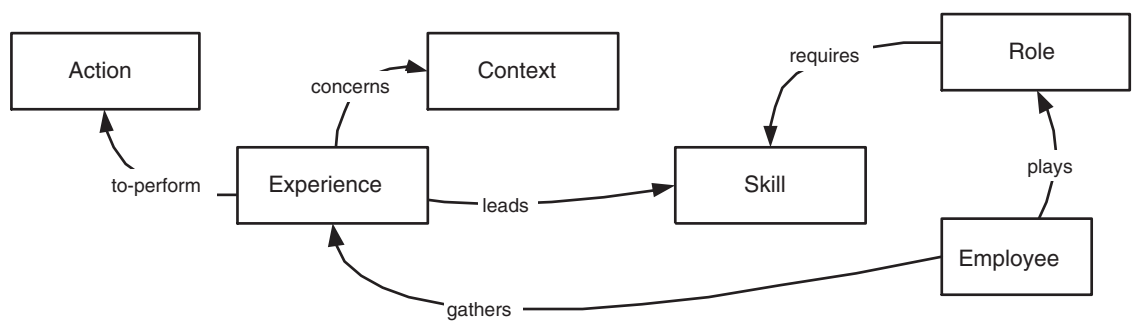

Fig. 3. Mobile employee gains required skills by gathering experience

it can encompass hint types built up from templates (similarly to the generic query templates in DECOR) as well as a retrieval of free-text notes entered by expert users and of documents accessible in organisational repositories. One of the central threads of work within the Pellucid project is the development of an ontology of the hints themselves, which is a basis for linking them with a representation of the work process.

The active hint is a formal representation of organisational experience (see Fig. 2). The example active hint is presented below:

Context: Creating a quarterly report

Action: Use

Resource: QuarterlyReportTempl.doc

Justification: It is a template

\section{$5 \quad$ Mobility Model}

A mobile employee changes his/her position within the organisation as a normal progress of his career. Each position requires some skills. Accomplishment of tasks at the particular position or in the scope of the particular role leads to a new experience accumulation and consequently to the skills (cf. Fig. 33).

It is important to note that most of skills are common for all roles within the organisation. They could correspond to the nomenclature, documenting techniques, procedures, etc. For this reason an employee who is changing position cannot be treated as a novice. A mobile employee should not be bothered with hints concerning the skills already possessed. The system should recognise current needs of the employee and provide only suggestions relevant to the employee's experience and skills [23].

\section{Domain and Context Modelling}

Trying to define the domain for each of Pellucid pilot applications it is necessary to find a general and simple criterion that could be used to model other applica- 
tions which follow a defined workflow. In this section we explain our approach to describe the domain according to two aspects: the procedure and the dossiers.

Indeed, at the first level of approximation we can see the domain as a space (the dossier space) where dossiers are distributed along two axes:

1. the technical data description,

2. the procedure description.

Each dossier is represented by a point on the axis 1, its life is represented by a line parallel to axis 2 . However, each value on the two axes is represented by a multidimensional vector in the two spaces of a dossier description and a procedure description. This could be a general approach. From this point we started to model the two spaces. For each application, we identified some parameters that, in a univocal manner, characterize the procedure and the dossier. A point in this bi-dimensional space can therefore represent the characteristic part of a case, in case-based reasoning. The action, that leads to the subsequent point (the dossier fixed), is the lesson part. The context (cf. Fig. 21) is therefore described both by the procedure and the particular problem to face (the dossier), which, in turn, are described by a set of parameters that are dependent on the application. Therefore, we have a procedural part, that can inherit some general concepts about workflow and activities, and a more specific part which is described by very technical concepts. Thus, we simplified the problem of context modelling, by dividing it into a domain specific component and a generic one.

One of Pellucid pilot application is provided for the design and installation of traffic lights by the mobility and transport department of one municipality in Italy. In this pilot site procedural data consists of general information (e.g. activity status, notes written within an activity, documents attached to the activity, e-mails sent during each activity) and information related to each activity

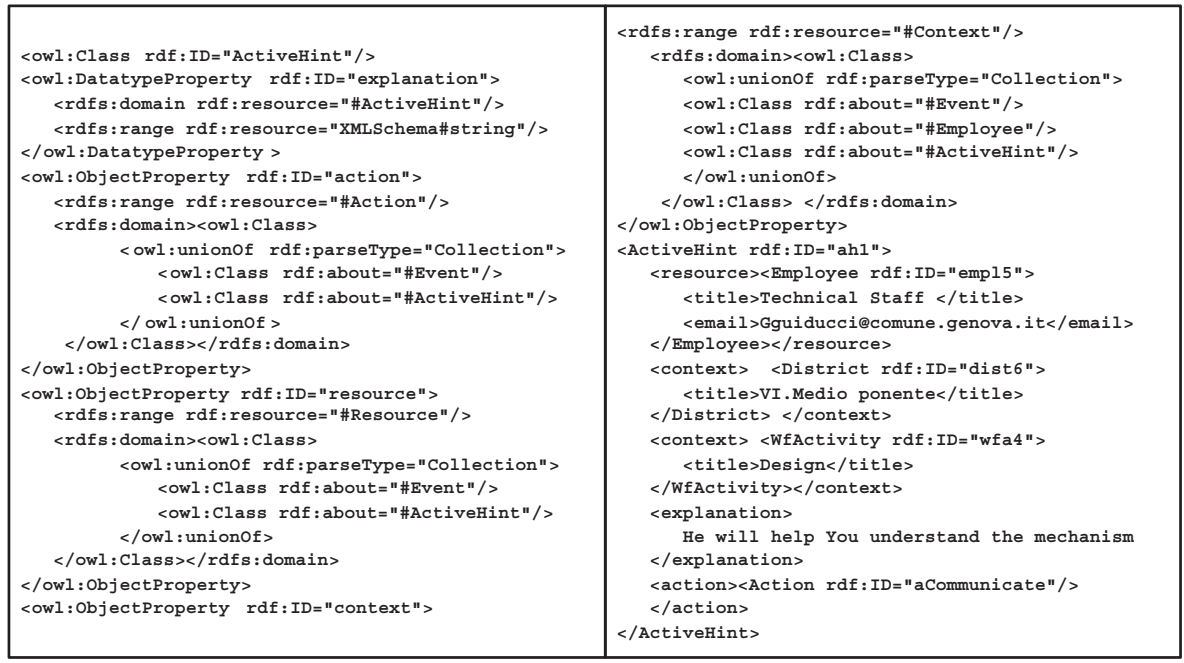

Fig. 4. Example ontology implementation. 
(e.g. input, preliminary analysis, data collection, design, installation, economical evaluation, external evaluation). Technical data describes the attributes of dossiers (e.g. type of request, applicant, dates, location, crossing shape).

\section{Model Formalisation - Ontology}

Ontologies can be represented using UML standard, in any object oriented language (OOL) or in special ontology languages such as DAML+OIL[24] or OWL 25]. DAML, DARPA Agent Markup Language is developed by DARPA organization. One of the $\mathrm{W} 3 \mathrm{C}$ activities is to create a standard for ontology description - just OWL has been created, which started out from DAML+OIL. DAML+OIL and OWL are based on XML and RDF, thus ontology represented by DAML+OIL or OWL is easier to integrate with existing commercial technologies. The ontology represents structure of knowledge. The knowledge itself is mainly a set of instances of ontology elements, which need to be stored and manipulated. When representing ontology by OOL we need to store knowledge into object oriented database. Ontology represented by OWL can be stored in XML-based databases. OWL is quite easily readable by humans and computer system. XML parsers are available in any development environment. XML interfacing with other systems can be done easily based on XML-RPC or SOAP technology. Presentation of knowledge to the user can be done by using XSLT which transform OWL/XML data to HTML. OWL ontology can be manipulated by Java based Jena library 26] which provides several storage models, methods for ontology manipulation, RDF manipulation and query engine for RDF query language (RDQL).

When developing an ontology we can have two types of relation between entities: inheritance ("is a") and properties ("has"), thus we need to simplify the real relation from CommonKADS model to inheritance, properties and instances.

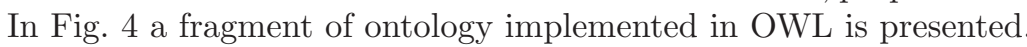

Implementation. The prototype of the Pellucid system was pre-evaluated and found very promising for the future users. We have got a lot of feedback, which is used for the refinement of some techniques and solution applied.

\section{Experience Gathering}

In the Pellucid framework, experience management is organised around three phases: capture, capitalisation and return phases. The capture phase is essential for the experience management because it enables gathering of experience and ensures the growth of the memory. Moreover, an enrichment of the Organisational Memory (OM) is a fundamental aspect when Experience Management aims to knowledge learning/maintenance. Thus, the objectives, the channels and the involved actors of the capture phase are outlined below. 
Objectives. Employees' experience captured in the Pellucid system (cf. Fig. 31 is twofold: explicit and tacit. Explicit experience is formal knowledge found in manuals, documentation, etc. Tacit or implicit experience is personal contextspecific knowledge, which results from human intelligence and consists of the mental representations or models (cognitive dimension) and skills and expertise of employees (technical dimension). As shown in 27, four conversions have been defined to describe the interactions between tacit and explicit knowledge. The capture phase was focused mainly on two of them: the externalisation, i.e. the "tacit-explicit" conversion and the combination, i.e. the "explicit-explicit" conversion. In our approach, the experience gathering stage will allow:

- to transform tacit into explicit experience by means of a human process. The externalisation will provide active hints (cf. Figs. 2).

- to capture explicit experience (such as a document or a potential contact) by means of an automatic process,

- to capture explicit knowledge (such as a contact) by means of a human process and more precisely by combination,

Experience Gathering Channels. The capture phase deals with experience collected during different activities of the employees. In Pellucid, only the individual experience is considered. Hence, the approach focuses on those gathering channels that are designed for individual use as opposed to those designed for group use. Two types of process will support these different channels: automatic and human processes.

In the case of the automatic process, i.e. through the workflow tracking system, two channels are identified: the channel enabling the capture of the documents and the channel enabling the capture of the potential contacts. The potential contact is a description of an actor, which is currently working on the workflow. In the case of the human process, three channels can be identified: the channel enabling the capture of contacts, the channel enabling the capture of active hints and the channel enabling the capture of indexing elements. The last one is key channel for the OM enrichment. The channel allows the user to define and underline some aspects of experience by using pre-defined elements of description such as keyword, tags, or sentences.

Actors. The concept of an actor refers to the principal types of users. Two types of actors are taken into account in the capture phase: the experienced employee regarded mainly as an actor enriching the organisational memory and the new employee. Some sub-types of new employees can be distinguished:

1. The novice: a new employee who has just graduated and is starting his career.

2. The skilled new-hire: a new employee who already has some experience on the same tasks, because had come from another company.

3. The relocated employee: a new employee who comes from the same company and therefore knows the internal procedures but has been previously involved in others tasks. 
Identification of employees' types at the experience gathering phrase is important to promote the evaluation feedback among the employees at the experience reuse phase (e.g. when a novice has evaluated a document as very good for his/her purposes, it is probable that this document is interesting for another novice).

\section{Conclusions}

In the paper, some results of development of experience management system for solving problems arising from high level of employees' mobility within organisations were presented. The concept of experience was modelled for the purposes of organisation. There was specified experience representation and some domain-specific applications. The approach to the experience gathering within organisation was introduced. Finally, implementation in form of ontologies was presented.

Acknowledgments. Thanks are due to European Commission and participants of the Pellucid project, IST-2001-34519. AGH-UST grant is also acknowledged.

\section{References}

1. Pellucid - A Platform for Organisationally Mobile Public Employees, EU IST2001-34519 Project, 2002, http://www.sadiel.es/Europa/pellucid/

2. Lambert, S., Stringa, S., Vianno, G., Kitowski, J., Słota, R., Krawczyk, K., Dziewierz, M., Delaître, S., Oroz, M.B., Gomez, A.C., Hluchý, L., Balogh, Z., Laclavik, M., Fassone, M., Contursi, V., "Knowledge management for organisationally mobile public employees", Proc. of 4th Working Conf. on Knowledge Management in Electronic Government, May 26-28, 2003, Rhodes, Greece, pp. 203-212.

3. Słota, R., Majewska, M., Dziewierz, M., Krawczyk, K., Laclavik, M., Balogh, Z., Hluchý, L., Kitowski, J., Lambert, S., "Ontology assisted access to document repositories in public sector organizations", Proc. of 5th Int. PPAM 2003 Conf., Sept. 7-10, 2003, Czȩstochowa, Poland, LNCS, vol. 3019 (in press)

4. Laclavik, M., Balogh, A., Hluchý, L., Słota, R., Krawczyk, K. and Dziewierz, M., "Distributed Knowledge Management based on Software Agents and Ontology", Proc. of 5th Int. PPAM 2003 Conf., Sept. 7-10, 2003, Czȩstochowa, Poland, LNCS, vol. 3019 (in press)

5. Abecker, A., Bernardi, A., Hinkelmann, K., Kühn, O., Sintek, M., "Toward a technology for organizational memories", IEEE Intelligent Systems, 13(3), pp. 40-48.

6. Reimer, U., Margelisch, A., Staudt, M., 2000, "EULE: A Knowledge-Based System to Support Business Processes", Knowledge-based Systems Journal, Elsevier, 13(5), pp. 261-269.

7. "DECOR: Delivery of Context-Sensitive Organizational Knowledge", project outline at http://www.dfki.uni-kl.de/decor/deldec/D1-Final.pdf.

8. Abecker, A., Bernardi, A., van Elst, L., Lauer, A., Maus, H., Schwarz, S., Sintek, M. "FRODO: A framework for distributed organizational memories. Requirements analysis and system architecture", DFKI GmbH, 2001.

9. Abecker, A., Bernardi, A., Hinkelmann, K., Kühn, O., Sintek, M. "Context-aware, proactive delivery of task-specific knowledge: The KnowMore project", International Journal on Information Systems Frontiers, 2000, 2(3/4), pp. 139-162. 
10. Gruber, T. R., A translation approach to portable ontology specifications", Knowledge Acquisition, Vol. 5 No. 2, pp. 199-220.

11. Benjamins, V.R., Fensel, D., Gómez Pérez, A., "Knowledge Management Through Ontologies", Proc. of the 2th International Conference on Practical Aspects of Knowledge Management, October 1998, Basel, Switzerland.

12. Abecker, A., Mentzas, G., "Active knowledge delivery in semistructured administrative processes", 2nd Workshop on Knowledge Management in Electronic Government, May 22-24, 2001, Siena, Italy

13. Staab, S., Schnurr, H.P., "Smart Task Support through Proactive Access to Organizational Memory", Knowledge-based Systems, Elsevier, Vol. 13 No. 5, 2000, pp. 251-260.

14. Van Kaathoven, R., Jeusfeld, M., Staudt, M., Reimer U., "Organisational Memory Supported Workflow Management", Electronic Business Engineering, Physica Verlag, 1999, pp. 543-563.

15. Koch, M., "Knowledge Management and Knowledge Agents in Campiello", Proc. of Workshop on Intelligent Agents in CSCW, 1998, Dortmund, Germany, pp. 44-52.

16. Bergenti, F., Poggi, A., Rimassa G., "Agent Architectures and Interaction Protocols for Corporate Management Systems", Proc. of ECAI Workshop on Knowledge Management and Organisational Memories, Aug 2000, Berlin, Germany, pp. 39-47.

17. Chen, J.R., Wolfe, S.R., Wragg, S.D., "A Distributed Multi-Agent System for Collaborative Information Management and Sharing", Proc. of 9th ACM International Conference on Information and Knowledge Management, November 6-11, 2000, McLean, VA USA, pp. 382-388.

18. KRAFT http://www.csc.liv.ac.uk/ kraft/

19. CORBA http://www.omg.org.

20. DCOM http://www.microsoft.com/com/default.asp.

21. Voyager http://www.objectspace.com

22. Schreiber, G., Akkermans, H., Anjewierden, A., de Hoog, R., Shadbolt, N., van de Velde, W., Wielinga, B., "Knowledge Engineering and Management: The CommonKADS Methodology", MIT Press, 2000.

23. Krawczyk, K., Majewska, M., Dziewierz, M., Słota, R., Balogh, Z., Kitowski, J., Lambert, S., "Reuse of Organisational Experience Harnessing Software Agents", The Agent Days, ICCS 2004, June 7-9, 2004, LNCS (in press).

24. DAML - DARPA Agent Markup Language, http://www.daml.org/

25. Web-Ontology (WebOnt) Working Group, "Web Ontology Language - OWL", 2003, http://www.w3.org/2001/sw/WebOnt/

26. Jena - A Semantic Web Framework for Java, http://jena.sourceforge.net/.

27. Nonaka, I., Konno., N., "The concept of "ba": Building a foundation for knowledge creation", California Management Review, 1998, 40(3), pp. 40-54. 